\title{
A Dynamic Scheme Based on Clustering for Wireless Sensor Networks MAC Protocol
}

\author{
BAO Li-yong, ZHAO Dong-feng, ZHAO Yi-fan \\ School of Information Engineering, Yunnan University Kunming, 650091, China
}

\begin{abstract}
Wireless Sensor Networks(WSNs), as one of the most important techniques in the 21st century, receives a universal attention to the research on its key techniques.A solution is urgently expected to meet the higher-quality service requirements of various applications on the limited bandwidth of wireless networks. Based on the ideas of Priority-based polling transmission with high-efficiency, and dynamic self-adaptation to loading, this paper presents a new analysis model of MAC Scheme of wireless sensor networks, which has made possible the Cluster Header capable of differentiating the nodes of different priority levels under mixed services policy and which has improved the flexibility and adaptability of the system via adjustment of the switch-in service in three time of load change input. The theoretical model of this protocol is established with Markov chain and probability generating function. Mathematical analysis is made on the mean queue length and the mean inquiry cyclic time of the common queue and the key node. It turns out that the findings from theoretical analysis correspond well with those from simulated experiments.
\end{abstract}

Index Terms: WSNs;clustering;priority-based polling system; dynamic load adaptation

(C) 2012 Published by MECS Publisher. Selection and/or peer review under responsibility of the International Conference on E-Business System and Education Technology

\section{Introduction}

Wireless Sensor Networks, an extension of the traditional cable network, has penetrated into every aspect of military sensing, security, environmental monitoring, traffic surveillance, medical treatment, building and structures monitoring, even anti-terrorism, etc[1-2]. With the popularity of WSNs application, the flow of WSNs is on the rise, making inevitable that its tasks be changed from the single data transmission to a variety of approaches combing data, voice and video.

However, since the sensor nodes are battery driven and it is impractical to recharge the battery for so many nodes after deployment, energy efficiency has been a key concern in the research work of WSNs[3]. In many WSNs applications, the data gathering has the Quality of Services(QoS) requirements in terms of BER performance, end-to-end timeliness and reliability,etc. Therefore, to design the scheme with low energy consumption and flexible QoS provisioning is the guarantee for the efficient information gathering in WSNs[4]. According to the analysis of sensor nodes, the main sources of energy consumption are sensing, wireless

* Corresponding author.

E-mail address: lybao@ynu.edu.cn 
transmission and data processing. And the energy consumption caused by wireless transmission is much more than sensing and data processing. On the other hand, since most technologies in WSNs, such as data routing, distributed information processing, have the requirements for the wireless transmission. Wireless transmission technologies have been identified as the key technologies determining the energy consumption and QoS, which are also the basis for other technologies[5-6]. Therefore, in this thesis, the author carried out a deep and systematic research work on the energy efficiency and QoS guaranteeing by establishing Cluster-based MAC Scheme in WSNs.

Actually, the quality of WSNs service mechanism determines the quality of network service. Corresponding to these characteristics this dissertation proposes an algorithm for polling scheme based on clustering. Usually clustering is an important method for hierarchy control in WSNs and maps the dynamic topology onto a relatively fixed architecture with multi-hop to single-hop communication in a cluster. In the suggested algorithm Cluster Header $(\mathrm{CH})$ polls the active nodes in a polling table which is used to register node addresses and priorities in a cluster to guarantee a QoS scheme of differentiated traffic priorities. The polling scheme not only avoids collisions but also reduces energy consumption for the only services to active nodes[7]. Furthermore, priority-based services can save energy to a large extent.

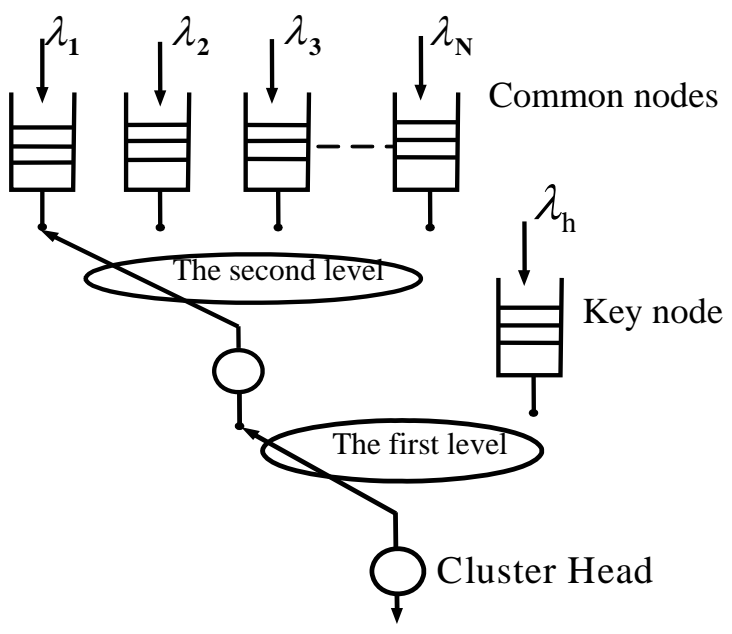

Figure 1. System Model

Based on queuing theory[8-9], this research established the two-class priority node gated polling system. The system is made up of one Cluster Header $(\mathrm{CH})$, one key node $h$ at the first level, and $N$ common nodes at the second level. The higher-priority key node CHplies an exhaustive-service policy with less time delay, which fits for the highly real-time performances. Meanwhile, the lower-priority common nodes use the gated policy with two-times services, which is based on the dynamically adjustable input load, to facilitate non-real-time transmissions. When the network load increases, service quality can be maintained with the increase of service times, which also fulfills the requirements of the non-real-time performances for fairness and time delay. The first and the second nodes receive alternatively the polling connection service from $\mathrm{CH}$. System model is shown in Fig.1

Markov chain and multi-dimensional probability generating function are applied to produce the systematic mathematical model, from which probability generating function of the system status variable is derived. Mathematical analysis and simulated experiment are made on the mean queue length and the mean cyclic time of the key node and the common nodes. 


\section{The Theoretical Model of the Protocol}

\section{A. The Operational Mechanism and Variable Definition of the System Model}

Polling services at each node include the following processes:

1) The $\mathrm{CH}$ polls at the common node of $i(i=1,2, \cdots, N)$ at the time of $t_{n}$. The lower priority business information packets number of queuing waiting for transmission in the buffer at the $i$ node is $\xi_{i}(n)$, when the high priority business information packets number of queuing waiting for transmission in the memory at the key node is $\xi_{h}(n)$. The $\mathrm{CH}$ provides transmission service for the $i$ node where the information packets in its queue is completed at the time of $t_{n 1}$. Then, if there is new information packets coming into the memory of the $i$ node within the service time, the lower priority business information packets number of queuing waiting transmission in the memory at the $i$ node is $\xi_{i}(n 1)$, and the higher priority business information packets number of queuing waiting for transmission in the buffer at the key node is $\xi_{h}(n 1)$. According to the service regulations, the server continues to work for the $i$ node, completing information packets within its queue via gated service for the second time in the $i$ node at the time of $t_{n 2}$. Likewise, if there is new information packets at the second gated service time, the server continues to provide transmission service for the $i$ node, completing information packets within its queue via gated service for the third time in the $i$ node at the time of $t_{n 3}$. At this time, the lower priority business information packets number of queuing waiting transmission in the memory at the $i$ node is $\xi_{i}(n 3)$, and the higher priority business information packets number of queuing waiting for transmission in the buffer at the key node is $\xi_{h}(n 3)$.The overall time of the CH's service of the $i$ lower priority queue is $v_{i}(n)$, with the information packets number of $\eta_{j}\left(v_{i}\right)$ when entering the $j$ node $(j=1,2, \cdots N, h)$ at the time of $\eta_{j}\left(v_{i}\right)$.

2) After the three-times gated service and via the transformational time of $u_{i 1}(n)$, the inquiry starts for the information packets queue waiting to be transmitted at the key node $h$, with the information packets number of $\mu_{j}\left(u_{i 1}\right)$ when entering the $j$ node $(j=1,2, \cdots N, h)$ at the time of $u_{i 1}$.

3) The key node $h$ receives service at the time of $t_{\bar{n}}$, when the higher priority business information packets number of queuing waiting for transmission in the buffer at the key node is $\xi_{i h}(\bar{n})$. After the completion of information grouping transmission within the key node via exhaustive service policy, the time of the server's service of the higher priority queue at the key node $h$ is $v_{h}(n)$, with the information packets number of $\eta_{j}\left(v_{h}\right)$ when entering the $j$ node $(j=1,2, \cdots N, h)$ at the time of $v_{h}$.

4) After the service of the key queue, there is a transformational time of $u_{i 2}(n)$. Transmission service comes to the $i+1$ common node at the time of $t_{n+1}$, with the information packets number of $\mu_{i}\left(u_{i 2}\right)$ when entering the $j$ node $(j=1,2, \cdots N, h)$ at the time of $u_{i 2}$. Likewise, the lower priority business information packets number of queuing waiting for transmission in the buffer at the $i(i=1,2, \cdots, N)$ node is $\xi_{i}(n+1)$ at the time of $t_{n+1}$.

\section{B. The Operational Conditions for the System}

According to the operational process of the system, the operational conditions are defined as follows:

1) The information packets entering each common node follow the independent and identical distribution of probability, with the distributional probability generating function and mean value respectively as $A(z)$ and $A^{\prime}(1)=\lambda$, and with the probability generating function and mean value of the information packets arriving at the key node h respectively as $A_{h}(Z)$ and $A_{h}^{\prime}(1)=\lambda_{h}$;

2) The time used by an information packets in the buffer of the common node when the server transmits services follows an independent and identical distribution of probability, with the distributional probability generating function and mean value respectively as $B(z)$ and $B^{\prime}(1)=\beta$, and with the distributional 
probability generating function and mean value of the transmission service time at the key node $h$ respectively as $B_{h}(z)$ and $\beta_{h}=B_{h}^{\prime}(1)$;

3) The random variable of the inquiry transformation time when polling from the $i$ common node to the key node $h$ follows an independent and identical distribution of probability, with the distributional probability generating function and mean value respectively as $R_{i 1}(z)$ and $R_{i 1}^{\prime}(1)=\gamma_{i 1}$. The random variable of the inquiry transformation time when polling from the $i+1$ common node to the key node $h$ follows an independent and identical distribution of probability, with the distributional probability generating function and mean value respectively as $R_{i 2}(z)$ and $R_{i 2}^{\prime}(1)=\gamma_{i 2}$;

4) The random variable of the time for the exhaustive service of information grouping arriving at the key node at any time slot follows an independent and identical distribution of probability, with the distributional probability generating function as $F_{c}(z)$;

5) The system operates on the FCFS rule. There is enough capacity of the memory at each node so that information grouping will not get lost.

\section{Probability Generating Function of the System Status Variable}

The system remains stable in the condition of $\sum_{i=1}^{N} \rho_{i}+\rho_{h}<1(\rho=\lambda \beta)$. The system status can be described with the Markov chain which is non-periodic and ergodic.

According to the operating process of the polling system, the probability generating function of the system status variable is shown as follows:

at the time of $t_{n 3}$

$G_{i 3}\left(z_{1}, z_{2}, \cdots, z_{N}, z_{h}\right)=\lim _{t \rightarrow \infty} E\left[\prod_{i=1}^{N} z_{i}^{\xi_{n 3} 3} z_{h}^{\xi_{n 3}}\right]$

$=G_{i 2}\left(z_{1}, z_{2}, \cdots, B_{i}\left(A_{h}\left(z_{h}\right) \prod_{j=1}^{N} A_{j}\left(z_{j}\right)\right), z_{i+1}, \cdots, z_{N}, z_{h}\right) \quad i=1,2, \cdots, N$

at the time of $t_{n 2}$

$G_{i 2}\left(z_{1}, z_{2}, \cdots, z_{N}, z_{h}\right)=\lim _{t \rightarrow \infty} E\left[\prod_{i=1}^{N} z_{i}^{\xi_{n 2}} z_{h}^{\xi_{n 2}}\right]$

$=G_{i 1}\left(z_{1}, z_{2}, \cdots, B_{i}\left(A_{h}\left(z_{h}\right) \prod_{j=1}^{N} A_{j}\left(z_{j}\right)\right), z_{i+1}, \cdots, z_{N}, z_{h}\right) \quad i=1,2, \cdots, N$

at the time of $t_{\bar{n}}$

$G_{i h}\left(z_{1}, z_{2}, \cdots, z_{N}, z_{h}\right)=\lim _{t \rightarrow \infty} E\left[\prod_{i=1}^{N} z_{i}{ }^{\xi_{i}^{(\bar{n})}} Z_{h}{ }^{\xi_{h}^{(\bar{n})}}\right]$

$=R_{i 1}\left(A_{h}\left(z_{h}\right) \prod_{j=1}^{N} A_{j}\left(z_{j}\right)\right) \cdot G_{i 2}\left(z_{1}, z_{2}, \cdots, B_{i}\left(A_{h}\left(z_{h}\right) \prod_{j=1}^{N} A_{j}\left(z_{j}\right)\right), z_{i+1}, \cdots, z_{N}, z_{h}\right) i=1,2, \cdots N$

at the time of $t_{n+1}$ 
$G_{(i+1) 1}\left(z_{1}, Z_{2}, \cdots, z_{N}, z_{h}\right)=\lim _{t \rightarrow \infty} E\left[\prod_{i=1}^{N} Z_{i}^{\xi^{(n+1)}} Z_{h}^{\xi^{(n+1)}}\right]$

$=R_{i 2}\left(A_{h}\left(z_{h}\right) \prod_{j=1}^{N} A_{j}\left(z_{j}\right)\right) \bullet$

$G_{\text {ih }}\left(Z_{1}, Z_{2}, \cdots, Z_{N}, B_{h}\left(\prod_{j=1}^{N} A_{j}\left(Z_{j}\right) F_{c}\left(\prod_{j=1}^{N} A_{j}\left(Z_{j}\right)\right)\right)\right) i=1,2, \cdots N$

D. The Analysis of Mean Queue Length

Given that $g_{i}(j)$ is the average number of information packets number at the $j$ node buffer when the $i$ node starts service at the time of $t_{n}$, then

$g_{i}(j)=\lim _{Z_{1}, \cdots, Z_{N}, Z_{h} \rightarrow 1} \frac{\partial G_{i}\left(Z_{1}, Z_{2}, \cdots, Z_{N}, Z_{h}\right)}{\partial Z_{j}}$

$i=1,2, \cdots N, h ; j=1,2, \cdots N, h$

According to the definition of queue length, the mean queue length is drawn via derived function algorithm as:

$g_{\mathrm{j} 1}(j)=\frac{\lambda_{j} \sum_{i=1}^{N}\left(\gamma_{i 1}+\gamma_{i 2}\right)}{\left(1+\rho_{j}+\rho_{j}^{2}\right)\left(1-\rho_{h}-\sum_{i=1}^{N} \rho_{i}\right)}$

and the mean queue length at the key node is:

$g_{\text {ih }}(h)=\lambda_{h}\left(\gamma_{i 1}+\gamma_{i 2}\right)+\frac{\lambda_{h} \rho_{i} \sum_{i=1}^{N}\left(\gamma_{i 1}+\gamma_{i 2}\right)}{1-\rho_{h}-\sum_{i=1}^{N} \rho_{i}}$

\section{E. The Analysis of the Mean Inquiry Cycle}

Based on the operational mechanism of the system and the theory of queuing, the mean inquiry cycle can be derived as:

$$
E\left[\theta_{i}\right]=\frac{\sum_{j=1}^{N}\left(\gamma_{j 1}+\gamma_{j 2}\right)}{1-\rho_{h}-\sum_{j=1}^{N} \rho_{j}}
$$




\section{Theoretical Calculation, Simulated Experiment and Analysis}

\section{A. Theoretical Calculation, Simulated Experiment}

Based on theoretical analysis, the simulated model of system is established. Both theoretical and simulated models are set in identical symmetrical conditions under which each node's information packets arrival follows the Poisson distribution with $\lambda$ as the parameter, and all the node parameters follow the distribution of identical laws. The length of information packets is 2700bits. The time slot width is taken as $10 \mu \mathrm{S}$. The time slot width is taken as $10^{\mu S}$. With $N=5, \quad \beta_{h}=\beta_{i}=10, \lambda_{h}=\lambda_{i}=\lambda, \quad \gamma_{i 1}=\gamma_{i 2}=2$, the system meets the stable condition of $\sum_{i=1}^{N} \rho_{i}+\rho_{h}<1$ in which M stands for the number of service times of the controlled gated service at common nodes. The data in the following charts show that the theoretical value of the system model in this research correspond well with that of the experiment and it can represent the first order feature of the system.

\section{B. Analysis of the System Feature}

Based on the justification of the rationality of the system model and system analysis, further analyses are made on the system feature:

1) From the system stability condition of $\sum_{i=1} \rho_{i}+\rho_{h}<1, \quad 0<\rho_{i}<1$ is inferred, signifying that when the gated service at common nodes becomes more frequent, the mean queue length diminishes and gated policy service or that with two or three times services exert influences on the mean queue length at the common nodes. The change of the mean queue length along with the arrival rate of information packets is illustrated in Fig.2. In case of the change with loading, stability and fairness as well as adaptability and flexibility of the system can be maintained via dynamic adjustment of gated policy service at common nodes.

2) The results from the calculations via Formulae (7) and (8) and the simulated experiments (Fig.3 and 4) all reveal that in the models of MAC protocol, the change in the times of service will not influence the mean queue length of key node and the system's mean cyclic time of the model.

3) Fig. 3 shows that the mean queue length of key node and that of the common nodes are clearly differentiated. Even when the arrival rate is high, the mean queue length of key node is smaller but more stable than that of the common nodes. Meanwhile, when the gated policy service at the common nodes increases, it still maintains a smaller value. The priority of the key node is thus preserved.

\section{Conclusion}

This improved polling scheduling strategy embodies the advantages of exhaustive and three-times gated policy service, and it classifies the system nodes by priority at two levels, based on which the dynamic adjustment of the comprehensive function of the system is made possible via the change of access connection so as to achieve the dynamic regulation of priority service along with the change in network loading and to enhance the fairness and flexibility of the WSNs system.

\section{Acknowledgment}

The authors would like to give their sincere thanks to the financial support by the Nation Natural Science Foundation of China(No.F0424104 and No.60362001). 


\section{References}

[1] Demirkol I, Ersoy C, Alagoz F, "MAC protocols for wireless sensor networks: a survey," IEEE Communications, vol. 44, pp. 115-121, 2006

[2] Akyildz I F, Melodia T, Chowdhury K R, “A survey on wireless multimedia sensor networks," The International Journal of Computer and Telecommunications Networking, vol.51 , pp. 921-960, 2007 .

[3] Lin Xiaohui, Yukwong Kwok, Wang Hui, "Cross-layer design for energy efficient communication in wireless sensor networks,”. Wireless Communications and Mobile Computing, vol.9, pp. 251-268, 2009,

[4] James M. Gilbert, Farooq Balouchi, "Comparison of energy harvesting systems for Wireless Sensor Networks, ” International Journal of Automation and Computing, vol.5,pp. 334-347,2008.

[5] Hnin Yu Shwe, JIANG Xiaohong, Suslginu Horiguchi, "Energy saving in wireless sensor networks," Journal of Communication and Computer, vol.6, pp. 20-27,2009.

[6] Rubin I, De Moraes L F. Message Delay Analysis for Polling and Token Multiple-Access Schemes for Local Communication Networks[J]. IEEE J. Select. Areas Commun., 1983, 1: 935-947.

[7] Takagi H. Analysis of Polling Systems[M]. Cambridge, MA: The M.I.T. Press, 1986.

[8] Zhao Dongfeng, Zheng Sumin, Message Waiting Time Analysis for a Polling System with Gated Service[J]. Journal of China Institute of Communications, 1994,15(2): 18-23. (in Chinese)

[9] Zhao Dongfeng, Zheng Sumin. Analysis of a Polling Model with Exhaustive Service[J]. Acta. Electronica Sinica, 1994, 22(5): 102-107. (in Chinese)

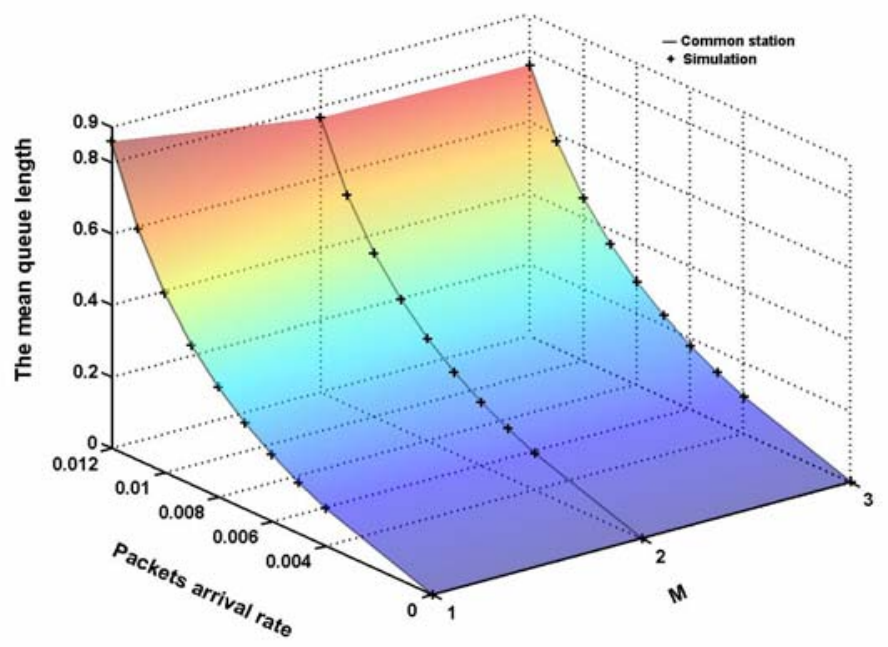

Figure 2. The mean queue length at the common nodes 


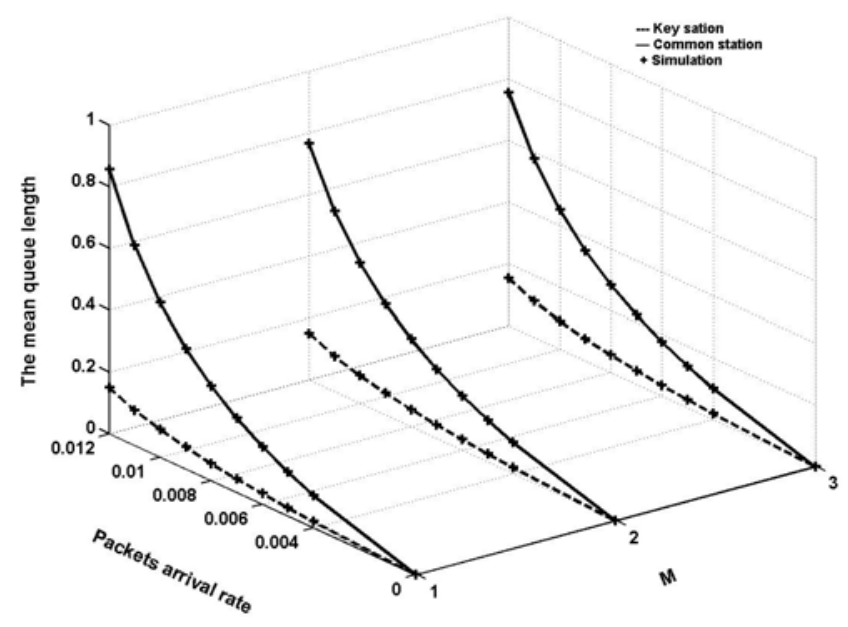

Figure 3. A contrast between the mean queue length of the key node and the common nodes

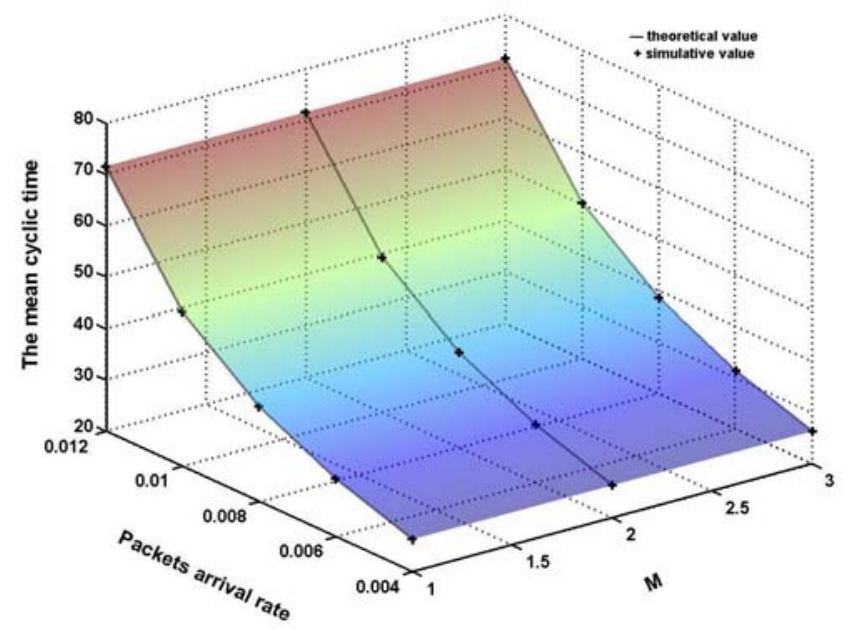

Figure 4. The mean cyclic time of the system 